\title{
A Frequency Ratio Account of Temporal Atomism
}

Author(s): Carey R. Carlson

Source: Process Studies, Spring-Summer 2021, Vol. 50, No. 1 (Spring-Summer 2021), pp. 107-127

Published by: University of Illinois Press

Stable URL: https://www.jstor.org/stable/10.5406/processstudies.50.1.0107

JSTOR is a not-for-profit service that helps scholars, researchers, and students discover, use, and build upon a wide range of content in a trusted digital archive. We use information technology and tools to increase productivity and facilitate new forms of scholarship. For more information about JSTOR, please contact support@jstor.org.

Your use of the JSTOR archive indicates your acceptance of the Terms \& Conditions of Use, available at https://about.jstor.org/terms 


\section{A Frequency Ratio Account of Temporal Atomism}

\section{Carey R. Carlson}

Carey R. Carlson is an independent researcher who studied the philosophy of science under Grover Maxwell at University of Minnesota. His recent book is The Mind-Body Problem and Its Solution (2nd ed.). Email: <carey .carlson1@gmail.com>.

ABSTRACT: This article examines the time duration of individual occasions in the light of the discovery that temporal succession produces frequency ratios. The frequency ratios are used to define energy ratios and the quantum. The manifold and the common particles are constructed graphically using the arrows of time, with the mass-ratios of the particles derivable from the graphs. The formal reduction of physics to time compels us to adopt Whitehead's conception of the physical universe as occasions of experience engaged in temporal/causal succession. The relative duration of the constituent occasions of the particles are determined by their graphs. In the final section, a refined account of the mind-brain interaction sequence confirms the duration of a human occasion as one tenth of a second.

\section{Introduction}

Alfred North Whitehead's Science and the Modern World provides a clue to the hard problem of mind and body. A sensory quality such as redness is no part of mathematical physics. The theoretical entities of physics owe their formulation to conjecture, while entities like redness belong to the immaterial dreamlike experience of sentient mind. Hence, the bifurcation of nature into "the conjecture and the dream." A solution is intimated in Russell's Human Knowledge. Russell contends that the physical world, including the 4-D manifold, is unperceivable, and that physics can at best discover the "causal skeleton" of the world, based on systematic conjecture as to the external causes of our phenomenal percepts.

The brain is in the head, but thoughts are not—so, at least, philosophers assure us. This point of view is due to a confusion between different meanings of the word "space." Among the things that I see at a given moment there are spatial relations which are a part of my percepts; if percepts are "mental," as I should contend, then spatial 
relations which are ingredients of percepts are also "mental." Naïve realism identifies my percepts with physical things; it assumes that the sun of the astronomers is what I see. This involves identifying the spatial relations of my percepts with those of physical things. Many people retain this aspect of naïve realism although they have rejected all the rest.

But this identification is indefensible. The spatial relations of physics hold between electrons, protons, neutrons, etc., which we do not perceive; the spatial relations of visual percepts hold between things that we do perceive, and in the last analysis between colored patches. (Russell 201-202)

When one realizes that Descartes's extension in space is unperceivable, one may grasp the idea of reducing dual-parameter space-time to a causal web of time-ordered events. That is a purely conceptual readjustment on the conjecture side of "the conjecture and the dream." Familiarity with Whitehead leads one to conclude that the role of sentient mind in the physical world is resolved by filling the causal web of events with Whitehead's occasions of experience.

For example, let the working hypothesis be that the ultimate realities are the events in their process of origination. Then each event, viewed in its separate individuality, is a passage between two ideal termini, namely, its components in their ideal disjunctive diversity passing into these same components in their concrete togetherness. There are two current doctrines as to this process. One is that of the external Creator, eliciting this final togetherness out of nothing. The other doctrine is that it is a metaphysical principle belonging to the nature of things, that there is nothing in the Universe other than instances of this passage and components of these instances. Let the latter doctrine be adopted. (AI 235-236)

Grover Maxwell shared my understanding, although he was not familiar with Whitehead. The present article is an attempt to add to Maxwell's view by claiming that the arrows of time form frequency ratios. I will show that the manifold and the common particles are produced by the simplest repetitive patterns that time can make.

Causal Set Theory and the Origin of Mass-ratio

Quantum theory is reconstructed using standalone causal sets. The frequency ratios inherent in causal sets are used to define energyratios, implicating the causal link as the quantum of action. Spacetime and its particle-like sequences are then constructed from causal links. A 4-D time-lattice structure is defined and then used to model 
neutrinos and electron clouds, which together constitute a 4-D manifold. A 6-D time-lattice is used to model the nucleons. The integration of the nucleus with its electron cloud affords calculation of the mass-ratio of the proton (or the neutron) with respect to the electron. Arrow diagrams, along with several ball-and-stick models, are used to streamline the presentation. ${ }^{1}$

I used "causal set theory" in the title of the above quotation so that physicists could relate my findings to an already established branch of physics. Causal set theory formalizes the discrete event ontology. The theory employs directed graphs to specify formations generated by a temporal/causal successor relation. Such formations are called "causal sets." The moments and transitions of time are termed "elements" and "causal links." Rafael Sorkin, who founded causal set theory, initially thought he had found the theory of everything. He asserted that causal sets satisfy special relativity "out of the box," that causal sets are just time, and that the world is a causal set. Before I ever heard of causal sets by name, I had employed the equivalent of causal sets and causal set graphs in The Mind-Body Problem and Its Solution. For documentation of my two main sources, I selected passages from Russell's Human Knowledge and Whitehead's Adventures of Ideas.

Einstein used the limiting velocity as an unexplained postulate to derive the rest of special relativity. There are fresh TED Talks online with physicists asserting that there is still no explanation for the limiting velocity. Causal sets explain the limiting velocity because any change of spacelike interval is a purely structural consequence of the stepping action of time. Thus, space cannot outrun time. The continuing pursuit of theories that do not explain the limiting velocity indicates the persistence of belief in instantaneous extension in space.

I am not a Whitehead scholar, and I am highly selective regarding Whitehead's ideas that I employ. However, his panpsychism was the incentive that led me to graphs of time and the frequency ratios formed. Once found, this discovery stands on its own merits without the support of Whitehead's interpretation. The discoveries are offered here for their effects on Whitehead scholarship, not the other way around. I will simply introduce the frequency ratios and follow the logical consequences in accord with the following prescription.

In framing a philosophic scheme, each metaphysical notion should be given the widest extension of which it seems capable. It is only in this way that the true adjustment of ideas can be explored. More 
important even than Occam's doctrine of parsimony-if it be not another aspect of the same-is this doctrine that the scope of a metaphysical principle should not be limited otherwise than by the necessity of its meaning. (AI 237)

\section{Temporal Succession and Its Frequency Ratios}

Was Whitehead a temporal atomist? The following passage strongly suggests that he was.

The notion of the contiguity of occasions is important. Two occasions, which are not contemporary, are contiguous in time when there is no occasion which is antecedent to one of them and subsequent to the other. A purely temporal nexus of occasions is continuous when, with the exception of the earliest and the latest occasions, each occasion is contiguous with an earlier occasion and a later occasion. The nexus will then form an unbroken thread in temporal or serial order. The first and the last occasions of the thread will, of course, only enjoy a one-sided contiguity with the thread. (AI 202)

In a continuous series, there is always another moment between any two, so with respect to any given moment, there is no next moment. Thus, no two moments are contiguous in a continuous series. Since Whitehead stresses that "the notion of the contiguity of occasions is important," I believe that Whitehead was a temporal atomist. It is unlikely he would be careless with the word "contiguity."

A discrete time series can be illustrated by a directed graph of arrows connected tail to head (see figure 1). Each node represents a moment of time and each arrow represents a discrete temporal transition from one moment to another.

In figure 2, the nodes of the diagram (the moments) can represent occasions of experience. The connecting arrows (the temporal transitions) then represent Whitehead's physical prehensions. Temporal succession is conflated with causal succession, so that a moment of time can have more than one successor and more than one predecessor. There are four ways that three moments can be connected by time steps. The graph on the far right of figure 2 is not a time graph, because each occasion is its own

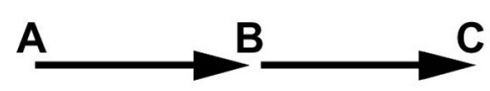

Figure 1. A Discrete Time Series. 

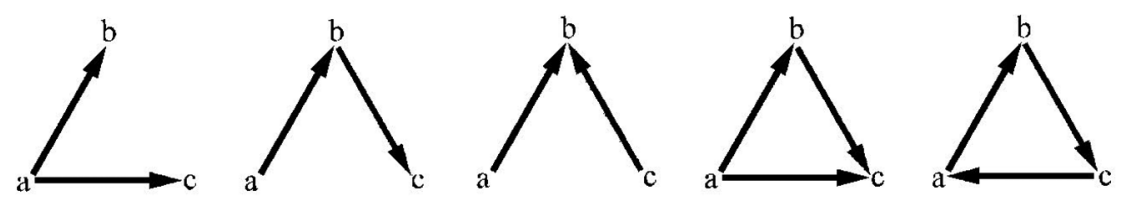

Figure 2. Time Graphs with Exactly Three Moments.

causal ancestor and its own causal descendant. No occasion can come before or after itself.

From left to right we see a fork in time, then a time series of two steps, then a convergence of time steps, and finally, a time triangle that features forking and convergence in combination to form a reconvergent sequence. The time triangle is the simplest graph to feature frequency ratios. It has a two-step path from $a$ to $b$ to $c$ and a one-step path from $a$ directly to $c$. Both paths begin at the same time and end at the same time. Thus, both paths span a single common time interval, which affords comparison of the frequencies of the two paths. One path takes two steps while the other path takes one step to traverse the common time interval, forming a frequency ratio of 2:1. Figure 3 is another graph that shows the frequency ratio $2: 1$.

There are three causal routes from the bottom event to the top one. Either side route involves two causal transitions with an intermediate event along the way. All three routes determine the same temporal interval, since they all begin at the same time and end at the same time. Either side route has twice the temporal frequency of the middle route.

Figure 4's alternate routes between common end points has two arrows forming one route and three arrows forming another, producing a frequency ratio of 2:3. Time diagrams exist for frequency ratios ranging across the rational numbers.

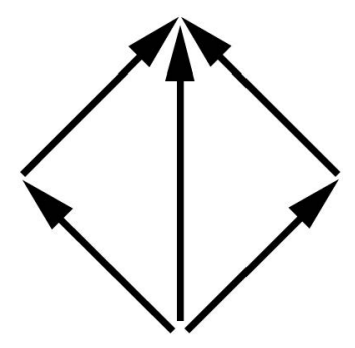

Figure 3. Frequency Ratio of 2:1.

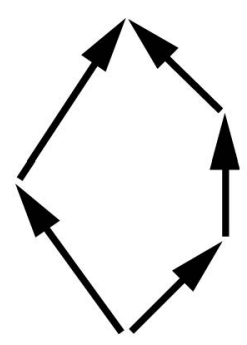

Figure 4. Frequency Ratio of 2:3. 


\section{Defining Energy in Terms of Frequency}

Planck discovered the coupling of energy and frequency at the quantum level, expressed in the formula $\mathrm{E}=\mathrm{hf}$. Thus, two energy values, $\mathrm{E} 1$ and E2, are equal to two frequency values, hfl and hf2. The ratio of two energy values, E1 / E2, is equal to the ratio of two frequency values, f1 / f2. Planck's constant drops out. The frequency ratios can define the energy ratios. The step of discrete time is the unit of the frequency ratios. Thus, the step of time is the quantum of the energy ratios. We have a structural definition of energy and its quantum in terms of time alone, illustrated in the simplest case by the time-triangle diagram of three arrows. Directed graphs can now serve as quantum schematics, each arrow depicting a quantum.

Notice that the physical prehension, not the occasion, is the unit of the frequency ratios and the quantum of the energy ratios.

\section{Relative Duration}

Doing away altogether with the dimensional units of physics, the metric of temporal succession will be based entirely on frequency ratios as shown by arrow diagrams. Only ratios will have absolute values. Empirical measurement always involves comparisons. Even the second of time is an arbitrary unit of duration. That is why a ratio-based metric is ideal for the theoretical foundation of physics.

The reciprocal of a frequency value is a time period or measure of duration. A quantum inherits the relative frequency value of the sequence to which it belongs. The reciprocal of a quantum's relative frequency is its relative duration. Higher frequency sequences consist of quanta with higher frequency and shorter duration than the quanta of lower frequency sequences. The physical prehension, or temporal transition, is the unit of frequency ratios. Thus, the prehension, which is the quantum, has the duration value. Judging from the arrow diagrams, the occasion has no duration of its own. We must include prehensions in order to gauge the lifetime of an occasion. Since an occasion "has its being" in its becoming and perishing, it makes sense to include its physical prehensions in the accounting of its duration.

\section{Space-Time Replaced by a 4-D Time Manifold}

The usual understanding of physical space is confined to geometric features, such as areas, volumes, points, and lines. These features can be ascribed to a person's visual experience, even during dreaming. Science 
must have a space that is consistent for all observers, a harmonization of perspectives based on waking perception, discarding the data of imagination and dreams. Furthermore, the space of physics must do without color or sensory qualities in its definition. It follows that we do not perceive physical space. We are restricted to conceiving it. The same holds true for the entities that occupy physical space. The whole apparatus of physics is refined from commonsense belief in a world not limited to our sensory experiences. Though we might be aware of the distinction between perceived space and the space of physical theory, we unhesitatingly borrow the geometric features of visual space and carry them over to the space of physics, as we do when learning Euclidean geometry in school. We fail to distinguish pure geometry, which is mathematics, from the geometry of our actual world, which is physics. The requisite distinction is found in the very purpose of science, which is to build a predictive causal framework for our scattered perceptions.

Einstein remarked that a discrete manifold could provide an inherent metric, but he never foresaw the discrete time manifold, much less the inherent frequency ratios it provides. The concept of a manifold created by sheer temporal succession is clarified by diagrams in which the arrow of time is the only graphic element. Figure 5 shows an extendable discrete time manifold of two dimensions. It serves to illustrate Whitehead's definition of contemporary events: "It is the definition of contemporary events that they happen in causal independence of each other. Thus two contemporary occasions are such that neither belongs to the past of

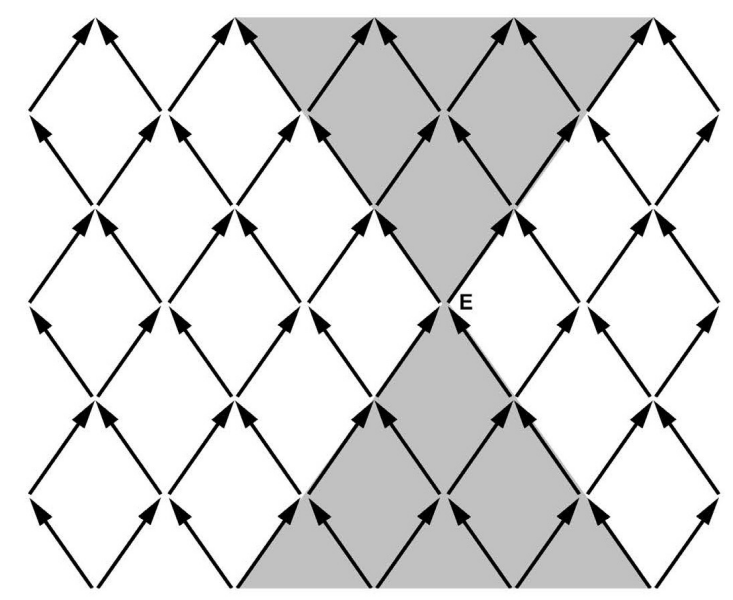

Figure 5. The Causal Past and Causal Future of E. 
the other. The two occasions are not in any direct relation to efficient causation" (AI 195). Occasion "E" in figure 5 has its own unique set of past and future occasions. Occasions within the unshaded regions are the contemporaries of $\mathrm{E}$. A contemporary of $\mathrm{E}$ is causally connected to $\mathrm{E}$ only indirectly via common causal ancestors or common causal descendants. Two contemporaries are "causal cousins." Neither is in the direct lineage of the other.

With Newtonian space, an instantaneous "now" sliced across the spatially extended universe. With special relativity, the "now" is proprietary to each occasion. The discrete time manifold illustrated in figure 5 has no primitive spatial relations to support instantaneous spatial extension. Temporal transitions alone suffice to form the manifold. In the passage quoted above, Whitehead refers to a "purely temporal nexus of occasions" when referring to a strictly serial nexus. Figure 5 depicts a nexus that is not purely serial, yet it is purely temporal.

Notice also, in figure 5, that if occasion E did not exist, then the four transitions that connect $\mathrm{E}$ to other occasions would not exist either. That would form a hole in the manifold, which is a legitimate alternative possibility. Attributing perfect uniformity to the manifold restricts the structural possibilities of temporal succession, ruling out options that are important for the reduction of physics to time.

The "hex cell," consisting of six moments connected by ten transitions, can be used to construct an extendable 4-D time manifold (see figure 6).
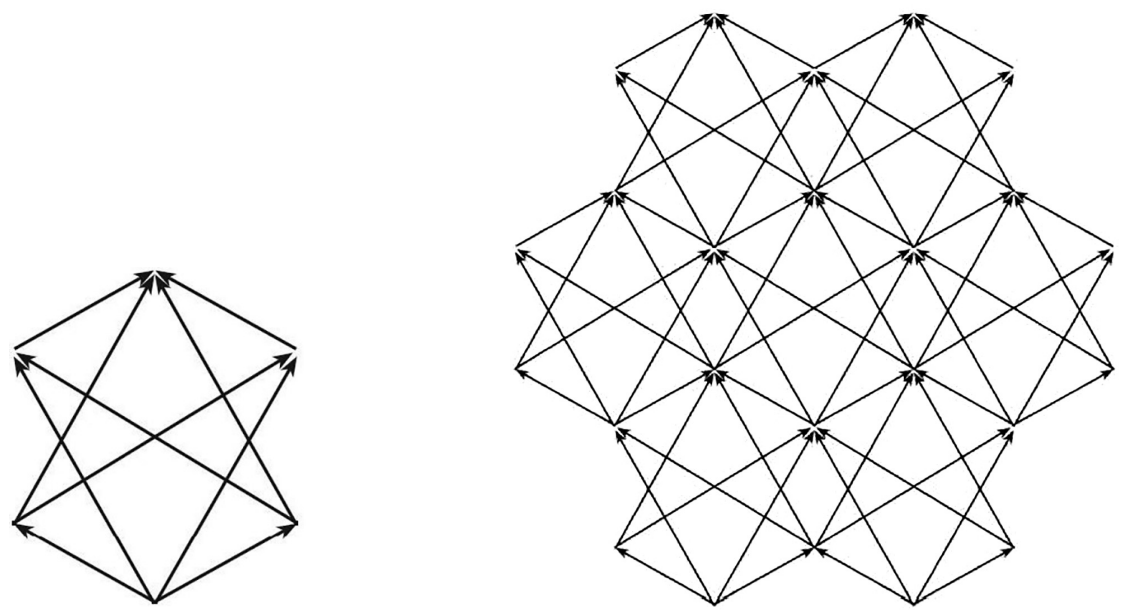

Figure 6. An Extendable 4-D Time Manifold. 
Each interior node has four arrows arriving and four departing, the defining characteristic of four dimensionality. The diagram can be outfitted with more hex cells to depict 4-D regions of any extent. Such a $4-\mathrm{D}$ region is made of quanta, like everything else in the theory of sheer temporal succession.

A "bounded region" can be defined as a region graphed by a "closed diagram," a diagram that has a single earliest moment and a single latest moment. Any closed diagram can be repeated as a cycle in chained repetition, such that the last moment of one cycle is the first moment of the next. By building up physics from closed diagrams, we confine ourselves to what can be confirmed by experiment. Any experiment is bounded by two moments- the triggering moment when the experiment is initiated and the final moment when the outcome is known. In between, nature enacts a causal sequence that results either in the predicted outcome or something else.

\section{The Reduction of Particles to Time}

The hex cell that was used to make a 4-D manifold will also serve, in chained repetition, to make an electron. The hex cell has locations for additional quanta that provide charge, momentum, and spin (see figures 7 and 8).
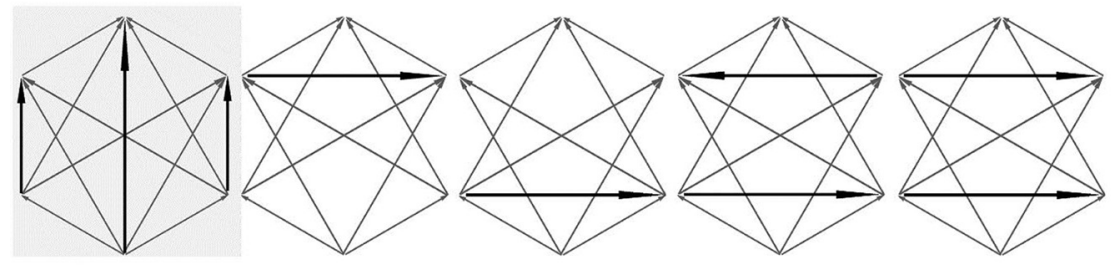

Figure 7. Quanta of Charge and Momentum.
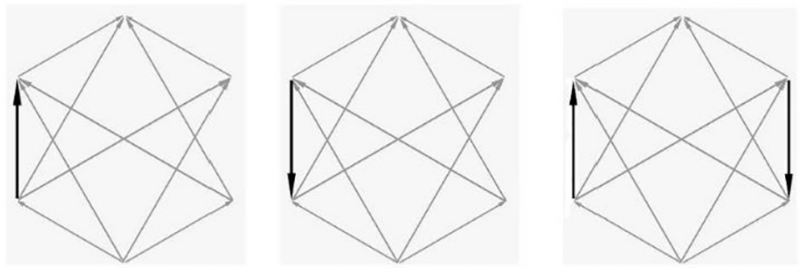

Figure 8. Spin Features. 
The vertical arrows represent quanta of forward momentum. The horizontal arrows, which necessarily break the bilateral symmetry of the cell, represent charge quanta. Spin options give the cell a type of "handedness." (The hex cell accommodates one "down arrow" at the most. Two down arrows would form a closed loop, violating chronology protection.)

A bare hex cell with no charge quanta may be called a "neutrino cycle" because it forms, in chained repetition, a neutrino. A hex cell with charge quanta may be called an "electron cycle" because it forms, in chained repetition, an electron.

\section{Bohr's Formula}

The following set of four sequences in figure 9 show a free electron, electron clouds of hydrogen and helium, and a cloud that absorbs and reemits a photon. The whole atomic series follows suit with these initial sequences in producing the discrete Bohr line frequencies for photon absorption and emission by the atoms.

I have drawn the hex cycles as neutrino cycles, without any charge quanta. As drawn, the sequences depict modes of neutrino propagation. If we populate the hex cycles with charge quanta, we get electron clouds. In that case, we would have, from left to right, a free electron, a hydrogen cloud, a helium cloud, and, finally, a hydrogen cloud sequence disturbed by an encounter with a photon. The first three diagrams mark the start of a progression that continues in step with the periodic table. The stable clouds feature uniform cycles of uniform frequency. The f-number above a stable sequence is the frequency of its cloud cycle repetition. The cloud disturbed by the photon exhibits a modulation of cycle pattern and frequency. The frequency of the photon, either incident or emitted, is the difference in frequency of the two cycles involved in the modulation. This scenario, generalized to the whole series of cloud possibilities, yields Bohr's formula for the frequency/wavelength values of photons absorbed and emitted by the entire atomic series of atoms.

A free electron is an electron cycle in chained repetition. Its frequency of repetition is " $\mathrm{fl}$ " as labeled in figure 9. Taking $\mathrm{fl}$ as the provisional unit of frequency, the wavelength of each cycle is also 1. Each sequence is scaled so that thirty-six hex cycles span the same height on the page, indicating that the four sequences elapse in the same amount of time. From the diagram, the wavelength of a hydrogen cloud cycle is four times that of the wavelength of a free electron cycle. The wavelength of a helium cloud cycle is nine times that of a free electron cycle. The wavelengths 1,4 , and 


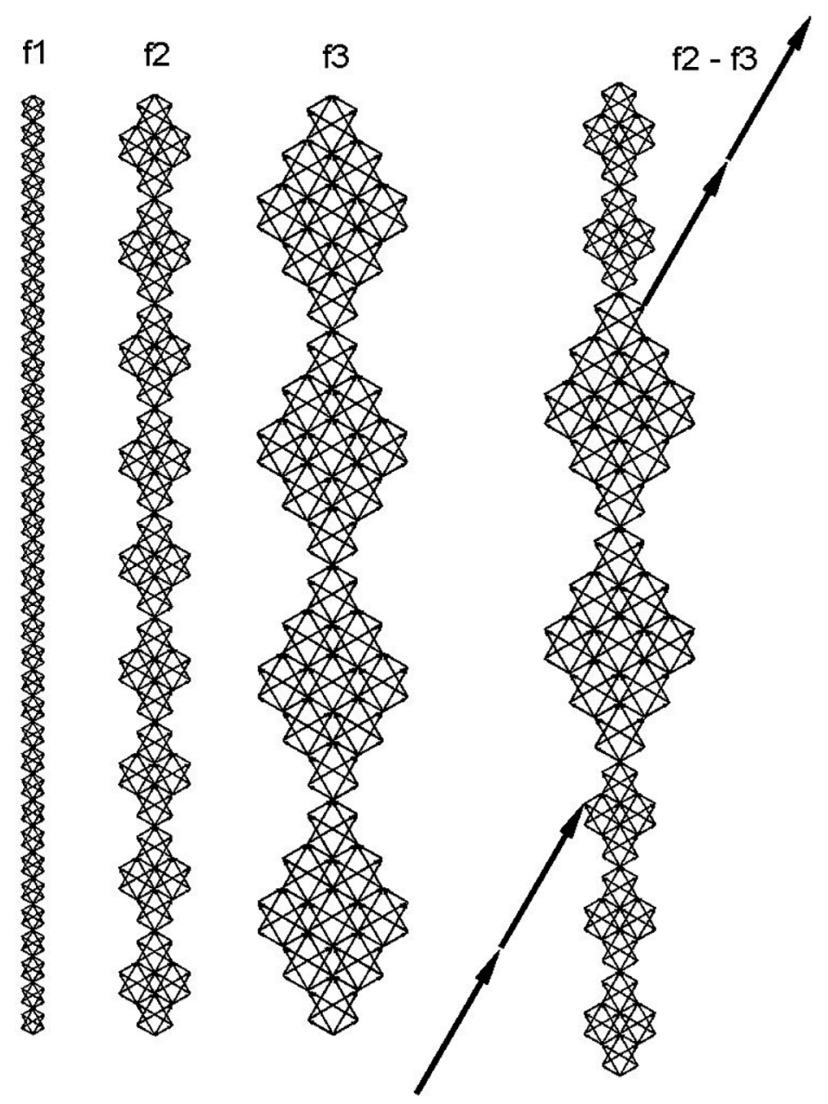

Figure 9. Graphical Account of Bohr's Formula.

9 correspond to frequencies $1,1 / 4$, and $1 / 9$, respectively. The intruding photon has the frequency $\mathrm{f} 2-\mathrm{f} 3$, or $1 / 4-1 / 9$. Bohr's formula for frequency, neglecting a constant scale factor, is $1 / \mathrm{k}^{2}-1 / \mathrm{n}^{2}$. For integer values $\mathrm{k}=2$ and $\mathrm{n}=3$, our graphical account gives the same photon frequency as Bohr's formula.

Notice that the electron as graphed has frequency and wavelength values. The wave-particle duality is resolved by means of discrete constructions. Even the electron clouds have their own frequency and wavelength values.

If we take the electron cycle as the unit of energy, then the progressive scaling that stretches thirty-six cycles to the same height on the page for each sequence means that the same amount of energy plays out in the 
same amount of time for each sequence. Thus, a type of symmetry is the basis of the discrete time dilation from one cloud sequence to the next. The discrete time dilation can serve as the basis for a discrete version of general relativity. General relativity uses the curvature of space-time to describe gravity, with time dilation being a consequence. An ad hoc postulate states that the amount of curvature is proportional to the local mass density. Assuming the nuclei dilate in step with their electron clouds, greater nuclear mass is tied to greater time dilation, explaining gravity with a symmetry principle rather than an ad hoc postulate.

Bohr's formula encapsulates a wealth of spectroscopy data. An explanatory model of the underlying structure of electrons and their behavior should not be more complex than the empirical data it is supposed to explain. A model represents theory, while Bohr's tidy formula represents the empirical data. We now have an account of electron-photon dynamics that is nearly as simple as the formula that encapsulates the data. We have no fundamental units of measure. We are left with the diagrams themselves, in which the electron appears as the real-time clock that sets the base frequency for the electromagnetic spectrum.

It is evident that four dimensionality arises at the smallest scale with the electron and neutrino. Electron clouds and neutrino formations share the telltale hex-cycle structure of the 4-D manifold, so we may speculate that they constitute what there is of the manifold. Gaps in the manifold delineate the locally propagating sequences of neutrino and electron formations. That would also explain the inverse square laws, tracing them to the inverse squares in Bohr's formula and the time dilation that appears in the graphical account.

\section{The Fine Structure Constant}

The fine structure constant is a dimensionless number, 137. Richard Feynman called it "the greatest damn mystery in physics." It is necessary for the calculations of quantum electrodynamics, but it is not the count of anything in his Feynman diagrams. However, 137 is the count of arrows in the time diagram in figure 10.

On the left in figure 10 is a region of 4-D lattice consisting of seventyeight arrows. Displaced to the right are the fifty-nine arrows that connect vertically aligned nodes (quanta in the proper time axis). The sum is 137 arrows exhibiting the fine structure of one cycle of the cloud of a helium atom. 

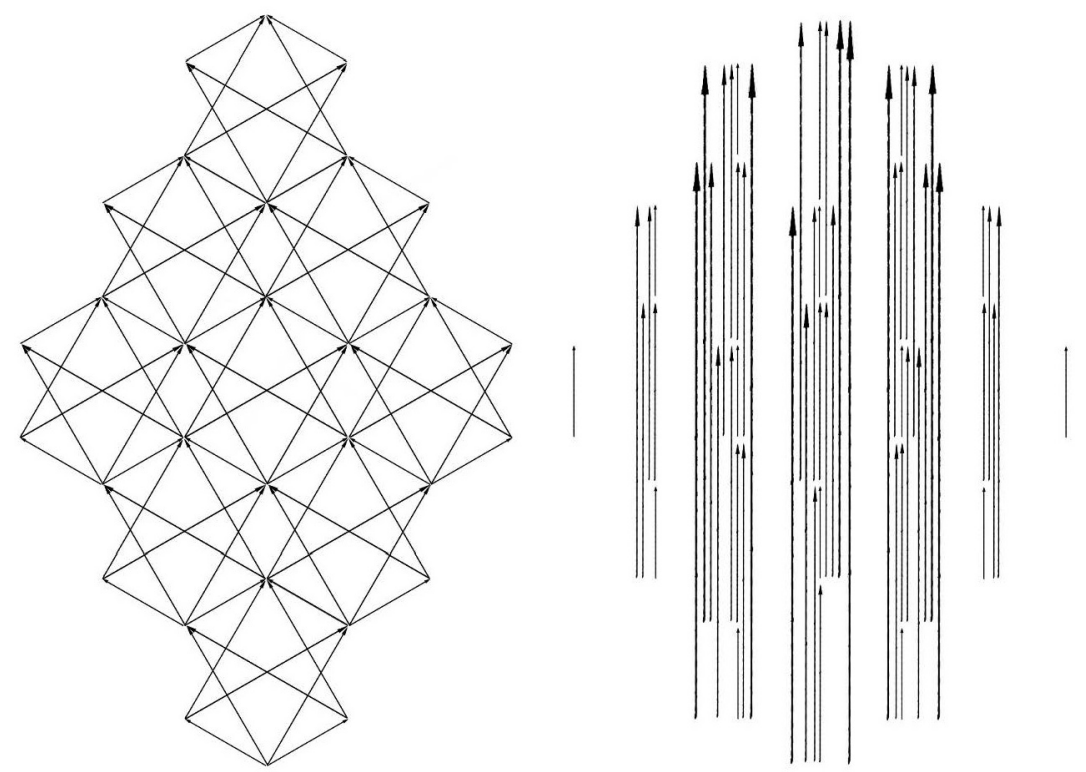

Figure 10.137 Arrows.

\section{The Nuclear Structure}

Attached to periodic nodes of an electron cloud sequence is a nuclear sequence composed of higher frequency quanta. This constitutes a synchronization of the nucleus to its electron cloud, assigning the nucleus its location in the 4-D manifold. The attachment of nucleus to cloud can only mean that the two discriminable sequences share periodic nodes.

\section{Summary of the Constructions}

Causal set constructions are relativistic by default, due to the exclusion of primitive spatial relations. Built from a successor relation, the theory has the same consistency as whole number arithmetic. The particle constructions describe self-organized patterns formed by temporal succession. Symmetry is the only organizing principle guiding the constructions, so symmetry assumes the role of the forces. Since symmetries reduce the complexity of a formation, the burden of explanation on physics is reduced to a minimum.

We are apt to think of the pace of human moments as "just right" for coherent experience. We are reluctant to grant such experience to 
subatomic occasions because of their nanosecond quickness. But there is no absolute measure of duration. A duration only has meaning in ratio comparison to other durations. The pace of experience is just right for the occasions of any sequence, regardless of its frequency ratio to other sequences.

Common sense realism regarding the existence of the physical world is not jeopardized by the reduction to time. The electrons and photons, the rock, the brain, the planets, and the galaxies all have their quanta and their mass energy. None of it has instantaneous spatial extension because the chaining of temporal transitions provides all the extensiveness there is. There is no such thing as a physical state of instantaneous organization. What exists all at once is only each individual moment. Since each moment is generic and primitive, there is no specification of its physical state. To refer to a "physical state" is to freeze out time and immerse oneself in the illusion of spatial extension.

Descartes made use of a piece of wax in his hand in order to bring his notion of physical existence into stark focus. The rock in my hand serves just as well as a piece of wax. My rock is made of quanta. The constituent quanta of the highest frequency connect to form the quark cycles. The quark cycles combine to form protons and neutrons, and these combine to form nuclei. The nuclei are interlaced with electron clouds to form complete atoms, which in turn combine to form molecules. The molecular patterns connect to form the rock. An arrow diagram of my rock would show how all its quanta are connected into a single elaborate sequence. The quanta are not undefined. Each quantum is an irresolvable step of time sequence. My rock is a propagating time sequence, made of temporal transitions from one moment to another. As it is with the rock, so it is with my hand that holds the rock, my body, physical objects in general, and the whole universe.

The key argument for panpsychism concerns the compatibility of human mental events with their immediate causes and effects. It has been a standing argument against mindlike entities that they are unlike the spatial entities of physics, so that causal interaction between the two is unintelligible. But now "the shoe is on the other foot." A time series of human moments is well suited to instantiate the causal order, as detailed in the final section. The remaining moments of physical theory have no specified attributes whatsoever. It is these latter moments that now stand in need of causal compatibility with mind. As for the causal pairing relations, since they connect mental event to mental event, they must be 
generally mental as well. To suppose otherwise would be to inject incompatibility for no reason.

\section{Issues between Whitehead and the Reduction of Physics to Time}

Hypothesis, speculation, conjecture-these roughly equivalent words express support for the essential role of hypothesis in theoretical physics.

Speculative Philosophy can be defined as the endeavor to frame a coherent, logical, necessary system of general ideas in terms of which every element of our experience can be interpreted. . . . Thus speculative philosophy embodies the method of the "working hypothesis." (AI 22)

When Whitehead argues for a special sense of causation, "non-sensuous perception," he is attempting to justify belief in the causal derivation of our sensory data without resorting to hypothesis. "We see with our eyes." But intervening between the eyes and the phenomenal percept are the optic nerves, which we do not perceive. Whitehead champions speculation anyway. "There is nothing in the universe other than instances of passage and components of those instances." The reduction to time puts the full weight of physics behind that speculation. We now have the "coherent, logical, necessary system of general ideas in terms of which every element of our experience can be interpreted." The coherence of the scheme provides all that one could want to justify the belief that there are external causes to our phenomenal sensory data.

Whitehead retained 4-D extension as a characteristic of occasions. This fails to deal with Descartes's extension in space as the defining characteristic of "the physical." It maintains "dualism all the way down." The patterns of temporal succession vary in their dimensionality: the one dimensionality of a light ray; the four dimensionality of the manifold; the six dimensionality of the nuclear structure. Occasions, depicted by nodes in the arrow diagrams, anchor all the relational patterns, but it is not the occasions that have the dimensions. Dimensionality is a structural feature of the patterns formed by temporal succession. If occasions do not have the physical attribute of extension, then there is no overlap of occasions, no "extensive abstraction." The next-to-next linking of occasions by causal inheritance relations, physical prehensions, is all that is needed to form the universe. Any of the arrow diagrams is an inheritance map, with each arrow depicting an inheritance passed from one occasion to a successor occasion. There is nothing physical to be passed from one occasion to 
another, because the theory of physics is already exhausted by the bare inheritance map itself. The form and content of subjective experience is all there is to an occasion, so the occasion has nothing else to give, nothing else to render to its successors.

\section{Smooth Sailing}

In the thirteenth century, the Buddhist philosopher Dogen formulated the idea of a causal web of mental events: "Mind is a knot in the net of inter-dependent origination." That is quite an accurate statement of Whitehead's theory of occasions. We now have the confirmation of this in physics. It is no accident that the fundamentals of physics can be derived from patterns of temporal succession. Here is a summary of what we have so far, as I wrote in a previous work:

In the light of the foregoing, let us reconsider the notion of the physical world described in Chapter 2. I said there that the meaning of "physical" amounts to an intuition of space and what's in it. Special Relativity implies that determinate location in space-time is due to time-ordering relations alone, as indicated by the arrow diagrams. The theory abandons purely spatial relations that define space as instantaneous extension. Since one event can have several immediate causal predecessors or successors, we must conceive the course of time as branched into locally separated streams. For physics, spacelike structure is part and parcel of this richer structure of time. The notion of space as an extended state is abandoned. This demands a corresponding revision to our conception of physical entities as inherently spatial. That conception belongs to a provisional stage of science that has been overturned by the discovery of a limiting velocity. The analysis of the universe into whole-and-part now finds the parts to be immaterial events that occur in somewhat regular patterns of succession. These patterns account for physical space, energy, shape, location and motion. This constitutes the de-materialization of matter and the dismantling of physical space required by Special Relativity. The meaning of "physical" therefore devolves upon events, their time relations, and the resulting patterns.... With the understanding that causal succession is equivalent to temporal succession, physics amounts to a theory of what comes before what. Progress in this theory has arrived at quantum events- discrete events which do not admit of further before-and-after analysis. These rudimentary events, and the time relation that orders them, are the quintessential physical entities. Our impression of the physical world as something substantial and immense owes to the sheer number of quantum events 
and their causal connections. If the subject of scientific investigation is simply the temporal sequence of events, then the physical world is not well characterized as "space and what's in it" but rather "time and what's in it." This has immediate consequence for the mindbody problem, since there is little difficulty in assigning the time of occurrence to a mental event. (Carlson, Mind-Body 73-75)

\section{The Frequency and Duration of Human Occasions}

Whitehead died before the cortical homunculi were discovered, and he never knew that temporal succession forms frequency ratios. By giving the discrete event ontology "the widest extension of which it seems capable" we can treat the brain as a purely sequential formation and describe in detail the causal location and functional role of human mental events in the brain (see Carlson, "Finite").

If one person's sentient mind is to be considered a discrete temporal series, the first order of business is to determine the frequency of the human series with respect to the other frequencies of physics. Psychophysical experiment indicates that $10 \mathrm{~Hz}$ is the frequency of human occasions. Let us consider the experimental data.

The closest physical correlate to human sentient awareness is the brain wave activity at the cortical surface, recorded by an electroencephalograph (EEG). In the late stages of amyotrophic lateral sclerosis (ALS), a patient can reach a locked-in stage, having lost the last vestige of voluntary motor control. The patient's mind is stranded, without even an eye blink to communicate to the outside world. In such a case, the brainwave activity indicated on an EEG is the only means by which a doctor can determine whether the patient's brain is still host to a sentient human mind. Dreamless sleep is indicated by slow rolling waves of several cycles per second. Alpha frequencies of $10 \mathrm{~Hz}$ accompany both the waking condition and active dreaming. The alpha frequencies are typically out of phase with one another, making a jumbled mess on the EEG record. A good meditator can bring the alpha frequencies into synchrony, producing a steady rhythm of coherent oscillation at $10 \mathrm{~Hz}$. That is one clue to the frequency of human occasions.

A stimulus probe on the visual cortex produces a spot in the subject's visual field. A pulsing stimulus, repeated at one location, produces a pulsing spot for the subject, until the pulse rate exceeds ten per second. As that stimulus frequency is approached, the sensory spot loses its frequency altogether and becomes steady. Testing the frequency response of the other 
sensory modalities reveals the same $10 \mathrm{~Hz}$ limitation. The implication is that we cannot register changes that are quicker than ten per second. The likely explanation: moments of human sentient awareness transpire at that rate and no faster.

A rate of ten-per-second for human moments is also appropriate to the delays involved in the conduction of efferent nerve signals from the brain to the muscles, and in the reverse direction, afferent signals from sense receptors to the brain. Reaction time, to avert a driving collision, for example, is not reducible to less than one tenth of a second. Reliable motor control of the body requires patience for the feedback, which is subject to the propagation delays of neural transmission. The human series is well qualified for central control of the human body, equipped at $10 \mathrm{~Hz}$ as the ideal frequency for the job.

Strobe lights at $10 \mathrm{~Hz}$ bother people, and epileptics are prone to seizure when they see such lights. All in all, given that we are seeking a finite frequency for the human series, a regular frequency of $10 \mathrm{~Hz}$ seems to be it. We are not aware of this frequency by introspection. It is ascertained only by reference to scientific hypotheses concerning a world that lies beyond the reach of anyone's introspective powers.

\section{Location of the Human Series in the Brain Sequence}

Brain scientists have mapped out a set of functional locations on the cortex called projection areas. These serve to pinpoint the location of the human series in the brain. The first two projection areas to consider are depicted by the motor homunculus and the sensory homunculus (see figure 11), which represent humanlike forms on the cortex that were first mapped out by Wilder Penfield. The topology of the human body is preserved in these shapes, but geometric distortions of the "little man" give him the appearance of a malformed fetus. You can stimulate the motor homunculus with a probe and get the corresponding part of the body to twitch into action, like operating a puppet. You can stimulate the sensory homunculus to shortcut the more remote stimulus that is normally needed on the surface of the body to achieve the same sensation. Each moment of a human series has additional predecessors and successors that belong to the brain, but not to the human series. Forking and convergence connect the human series to other cycles of the brain.

Quanta that fork off from the human series to the motor homunculus affect bodily movement. Quanta from the sensory homunculus converge 




Figure 11. The Cortical Homunculi.

upon the human series, updating the body image of somatic awareness. At cycles of $10 \mathrm{~Hz}$, the sequence of cause and effect is as follows:

- One human moment forks off, connecting to many moments of the motor homunculus.

- Effects are propagated along efferent nerve routes to the muscles.

- Muscle action causes feedback signals along afferent nerve routes to the sensory homunculus.

- Many moments of the sensory homunculus converge upon the next moment of the human series.

During the tenth of a second between the two bounding moments of the above cycle, one human quantum also transpires, propagating the human series. The spacelike equivalent of a tenth of a second is about 18,000 miles - plenty of range to gather concurrent influences from the surface of a cortex.

The standard conception of a brain is one of instantaneous extension in space, with no earlier and later involved in its composition. That is a brain without quanta. Such a brain has no place in our physics. According to special relativity, the cortical surface is a set of contemporaries-causal cousins related only by their causal ancestry. Such contemporaries are also poised to beget common causal descendants. The location of the mind in the brain is resolved by tracing the causal lineage of human mental events to and from the homuncular regions. The homunculi on the cortex are 
the key causal locators of human mental events. Other projection areas on the cortex have also been mapped out that correspond to other sensory fields of human phenomenology. Patterns of excitation at the retina are reproduced at the visual projection area. Auditory experience also has a patch of cortical surface devoted to it. A mental event typically involves all the sensory modes at once. The distinct phenomenal sensory modes correspond to the distinct patches of cortex devoted to the organs of sight, sound, and touch. As with the sensory homunculus, the visual and auditory projection areas are home to causal predecessors of the human series. From those cortical sites, the afferent system converges to a human percept, at which point the efferent phase of causal sequence is renewed.

Let us consider visual experience and its patch of cortex. In phenomenal vision, we have a spatially extended field of colored patches. The colorful visual field is part of a human mental event. As with color, we are directly acquainted with the inherent geometry of the visual field. We can judge with remarkable precision the size and shape of colored patches given in our visual experience. A good example is the extraordinary precision by which we can judge a rectangle to have height-width proportions of the golden mean. The ancients could judge with the same accuracy. It owes nothing to science. It is a type of ratio measurement that involves no scientific conceptions or assumptions. The visual projection area has a space-time metric based on the second as the standard unit of duration. The speed of light is a foot per nanosecond. Supposing the patch of visual cortex to be roughly circular and one inch in diameter, its spacelike extent is approximately one tenth of a nanosecond. The full spread of the subject's phenomenal visual field correlates to the full diameter of the cortical patch, so half the visual field corresponds to half the cortical patch, or one twentieth of a nanosecond. Proportionate size in the visual field is thus correlated to the metric unit of physics. This correlation is needed for an epistemological account of physical measurement, which requires sentient mental events in the laboratory and sensory data that are phenomenally given to them. The correlation of phenomenal measure to the nanosecond span of this or that cortical projection area is reliant on psycho-physical experiments. Perceivable sensory fields are correlated to the unperceivable domain of physics. We do not expand the domain of the perceivable by arriving at such correlations. In the case of hearing, a spacelike span across the auditory cortex correlates to an interval of phenomenal pitch. Psychophysics correlates the qualitative data of subjective experience to the conjectural model of theoretical physics. 
The human series has direct access to vision, hearing, and tactile information at the sensory projection areas. "Direct access" is unambiguous in our theory of physics. It means that select moments of the sensory projection areas are immediate causal predecessors of a human moment. Each such predecessor connects to the human moment by a single quantum. Likewise, direct action by a human moment upon some moment in the region of the motor homunculus means that a single quantum connects the human moment to the homuncular moment. The homunculi are situated on the cortical surface as if to provide convenient test points for a technician to troubleshoot the sensory and motor systems. In normal operation, they serve as staging areas for perception and control by the human series. The stable brain is a propagation of synchronized time cycles, featuring a wide range of frequencies and likely a wide variety of cycle topologies. The stability of human experience and its dependence on the brain means that the human series must be embedded in supportive cycles of $10 \mathrm{~Hz}$ frequency. These cycles provide a base of causal routine for the human series and connect it to the ladder of higher frequencies that constitute the rest of the brain.

\section{NoTe}

1. See http://vixra.org/pdf/1006.0070v1.pdf.

\section{Works Cited}

Carlson, Carey R. "Finite Eventism." Mind That Abides: Panpsychism in the New Millenium, edited by David Skrbina, Amsterdam, John Benjamins, 2009.

---. The Mind-Body Problem and Its Solution. 2nd ed., GoToPublish, 2019. Russell, Bertrand. Human Knowledge: Its Scope and Limits. 1948. New York, Simon and Schuster, 1967.

Whitehead, Alfred North. Adventures of Ideas. 1933. New York, Free P, 1967. 